Conclusion. Our observation of PLWH with venous thromboembolism sugges that this population has an increased risk of venous thromboembolism as compared to general population. Female gender is an independent risk factor for venous thromboembolism in PLWH and higher HIV viral load seems to associate with higher risk. Larger prospective studies in this population are needed to dissect the interplay between HIV and venous thromboembolism.

Disclosures. All Authors: No reported disclosures

\section{Weight Gain Associated With Antiretroviral Therapy}

Yesha Patel, $\mathrm{MD}^{1}$; Sheila Okere, $\mathrm{BS}^{2}$; Mark Lustberg, $\mathrm{MD}, \mathrm{PhD}^{2}$; Carlos Malvestutto, $\mathrm{MD}^{1} ;{ }^{1}$ The Ohio State University Wexner Medical Center, Columbus, Ohio; ${ }^{2}$ The Ohio State University College of Medicine, Columbus, Ohio

\section{Session: P-44. HIV: Complications and Special Populations}

Background. Obesity is a global public health crisis with a growing prevalence in persons with human immunodeficiency virus (PWH) population. In this study, we aimed to investigate factors associated with weight gain in the $\mathrm{PWH}$ population.

Methods. This was a single-centered, retrospective cohort study of our clinic patient population from January 1, 2015 to January 1, 2019. Patients with human immunodeficiency virus (HIV) were identified from the electronic health record and a randomized sample of 300 patients who had at least two follow up appointments, were on antiretroviral therapy, and had viral loads less than 200 were evaluated. The primary outcome was weight change over follow up. Cox Proportional Hazards models were used, taking a weight gain $>3 \mathrm{~kg}$ as the outcome, and the time on therapy between visits as the time to event. Robust linear regression was used to model mean changes in weight, accounting for influential observations. All analysis were performed in STATA 16.0.

Table 1

\begin{tabular}{|l|c|ccc|}
\hline & \multicolumn{2}{|c}{ Univariate model } & \multicolumn{2}{c|}{ Multivariate model } \\
& Difference \pm SE (kg) & p-value & Difference \pm SE (kg) & p-value \\
\hline Male gender & $1.38 \pm 0.61$ & 0.024 & $1.89 \pm 0.61$ & 0.002 \\
\hline Marijuana use & $-4.20 \pm 1.63$ & 0.01 & $-4.74 \pm 1.55$ & 0.002 \\
\hline Darunavir use & $-1.36 \pm 0.57$ & 0.016 & $-1.10 \pm 0.55$ & 0.046 \\
Rilpivirine use & $1.69 \pm 0.69$ & 0.014 & $1.97 \pm 0.66$ & 0.003 \\
\hline Etravirine use & $-3.62 \pm 1.50$ & 0.016 & $-3.08 \pm 1.43$ & 0.032 \\
\hline Bictegravir use & $4.54 \pm 2.11$ & 0.032 & $4.57 \pm 2.01$ & 0.024 \\
\hline White race & $-0.77 \pm 0.44$ & 0.10 & $-1.18 \pm 0.43$ & 0.007
\end{tabular}

Results. At baseline, $87 \%$ were male, $63 \%$ were white, and $65 \%$ were overweight obese. $30 \%$ were on a protease inhibitor, $46 \%$ were on non-nucleoside reverse transcriptase inhibitor, and $37 \%$ were on an integrase inhibitor. $64 \%$ were on Tenofovir disoproxil (TDF), $8 \%$ were on Tenofovir alafenamide (TAF), and $19 \%$ were on Abacavir. Mean weight change over follow up was significantly increased at $1.31 \mathrm{~kg}(95 \%$ $\mathrm{CI}=0.58-2.04 \mathrm{~kg}, \mathrm{p}=0.0004)$. TAF use and male gender were significantly associated with risk of weight gain $>3 \mathrm{~kg}$ in univariate analysis [respectively, $\mathrm{OR}=2.53,95 \%$ $\mathrm{CI}=1.30-4.92, \mathrm{p}=0.006 ; \mathrm{OR}=2.60,95 \% \mathrm{CI}=1.05-6.45, \mathrm{p}=0.04]$. In multivariate analysis, TAF use was significantly associated with weight gain $>3 \mathrm{~kg}$, while male gender was of borderline significance [respectively, $\mathrm{OR}=2.29,95 \% \mathrm{CI}=1.17-4.47$, $\mathrm{p}=0.01 ; \mathrm{OR}=2.40,95 \% \mathrm{CI}=0.96-5.97, \mathrm{p}=0.060]$. Significant factors associated with weight change are noted in Table 1.

Conclusion. As PWH are living longer on effective ARV therapy, monitoring for weight gain is required as obesity contributes to morbidity and mortality from cardiovascular disease and metabolic diseases. Key factors for weight gain in our clinic population include male gender, baseline diagnosis of hypertension, use of TAF, bictegravir use, and rilpivirine use.

Disclosures. Carlos Malvestutto, MD, Gilead Sciences (Advisor or Review Panel member)ViiV Healthcare (Advisor or Review Panel member)

952. Weight-Gain in Treatment Naive Newly Diagnosed HIV-Infected Persons After Initiation on Integrase Strand Inhibitor Based Treatment Regimens Zsofia Szep, MD ${ }^{1}$; Saishi Cui, $\mathrm{MS}^{2}$; Heather Conway, $\mathrm{MD}^{3}$; Alison Evans, $\mathrm{ScD}^{4}$; ${ }^{1}$ Drexel University College of Medicine, Philadelphia, PA; ${ }^{2}$ Drexel University School of Public Health and Epidemiology, Philadelphia, Pennsylvania; ${ }^{3}$ DarmouthHitchcock Medical Center, Darmouth, New Hampshire; ${ }^{4}$ Drexel University, Dornsife School of Public Health, Philadelphia, Pennsylvania

Session: P-44. HIV: Complications and Special Populations

Background. We performed a retrospective cohort study of treatment-naive patients with newly diagnosed HIV-infection enrolled at an urban university specialty clinic to identify whether INSTI-based ART regimens were associated with greater weight gain compared to NNRTI and PI based regimens in the first 12-18 months of treatment. The secondary aim of this study was to determine differences in weight gain between males and females within each of the three ART classes

Methods. Differences in weight change and BMI change were compared across ART class using nonparametric tests, specifically the Wilcoxon rank sum test Nonparametric tests were also used to compare differences in weight change and BMI change between males and females within each ART class. Data were analyzed using R Core Team, 2020

Results. Among the 348 individuals included in the study, $73 \%$ were African American and $79 \%$ were male and the median age was 32 years. There were 155 individuals initiating therapy on NNRTI based regimens (44\%), 58 were on PI based regimens $(17 \%)$ and 135 were on INSTI regimens (39\%). The median weight at baseline was $170.5 \mathrm{lbs}$. and the median body mass index was $25.4 \mathrm{~kg} / \mathrm{m}^{2}$. Median weight increased across all 3 ART regimens within the first 12-18 months of treatment. Median weight gain among the PI group was the greatest, at $6.8 \mathrm{lbs} .(\mathrm{p}=0.04)$. Median weight gain among the NNRTI group was the lowest, $.88 \mathrm{lbs}(\mathrm{p}=<.01)$. Median weight gain among those on INSTI based regimens was $4.8 \mathrm{lbs} .(\mathrm{p}=0.11)$. Among those on INSTI-based regimens, women had a greater median increase in weight compared to men, $10.1 \mathrm{lbs}$. compared to $3.2 \mathrm{lbs}$., $(\mathrm{p}=0.046)$.

Conclusion. Overall, among individuals initiating HIV treatment those initiating PI based regimens experienced the most weight gain and individuals initiating INSTI based regimens did not experience a significant weight gain. Women on INSTI based regimens did experience a significant weight gain in comparison to men.. More research is needed to elucidate specific ART regimens' causal role in weight gain and to identify risk factors for ART-associated weight gain.

Disclosures. All Authors: No reported disclosures

\section{Frailty Among People Living with HIV In Miami, A Cross Sectional Pilot Study}

Douglas Salguero, $\mathrm{MD}^{1}$; Jornan Rodriguez, $\mathrm{MD}^{2}$; Patricia Raccamarich, $\mathrm{MD}^{2}$; John Abbamonte, $\mathrm{PhD}^{2}$; Suresh Pallikkuth, $\mathrm{PhD}^{2}$; Allan Rodriguez, $\mathrm{MD}^{2}$; Savita Pahwa, $\mathrm{MD}^{2}$; Maria L. Alcaide, $\mathrm{MD}^{2} ;{ }^{1}$ Mount Sinai Medical Center, MIAMI Florida; ${ }^{2}$ University of Miami, Miami, Florida

\section{CFAR University of Miami}

\section{Session: P-45. HIV: Epidemiology and Screening}

Background. Frailty, a status of high vulnerability, is a clinical syndrome associated with adverse health outcomes and characterized by a constellation of various health deficits. Although age is a major contributor of being frail, HIV infection is associated with accelerated aging, and likely contributes to frailty. This association has seldom been evaluated. This study evaluated factors associated with frailty among PWH in Miami.

Methods. Cross-sectional study. Adults ( $>18$ years), HIV infected (HIV+) and uninfected (HIV-), virologically suppressed for at least 1 year $(<50$ copies/ $\mathrm{ml}$ ). Sociodemographic factors and the self reported FRAIL scale was administered (Fatigue, Resistance or ability to climb a single flight of stairs, Ambulation or ability to walk one block, Illnesses or non-HIV associated comorbidities, and more than $5 \%$ weight Loss in the previous year). Groups were categorized base on the FRAIL scale scoring as Non-Frail (0), Pre-Frail (1-2), and Frail (3 or more). The association by Frail categories were analyze using descriptive statistics and ordina logistical regression.

Results. N (40), median age was 43 years (SD 20.6); 35\% White; 20\% Hispanic; $52 \%$ females; 25 (62.5\%) HIV +/ 15 (37.5\%) HIV -. A small number of participants reported use of tobacco $2(5 \%)$ and alcohol $7(18 \%)$. More than half of the participants were frail or pre- frail (18 or $45 \%$ Non-Frail, 18 or $45 \%$ Pre-Frail, and 4 or $10 \%$ Frail, and HIV+ were more likely to be pre-frail or frail than HIV-, $72 \%$ vs $26 \%, p=0.019$ ). Frail scale symptoms were common among all participants but HIV+ reported higher fatigue than HIV- ( $85 \%$ vs $14 \%, \mathrm{p}=0.01)$. On Regression analysis, both HIV status and age were significant predictors of frailty status (HIV $\chi^{2}(1)=4.36, \mathrm{p}=.037$ and age $\chi 2(1)=13.48, \mathrm{p}<.001)$. When controlling for age, being HIV - on average reduced frailty by an odds of $2.16(\mathrm{~b}=-2.164, \mathrm{SE}=1.04, \mathrm{p}=.037,95 \% \mathrm{CI}[-4.2-0.13])$. When controlling for HIV status, for every one year of increase in age, the ordered log odds of being frail increased by $0.07(b=0.07, S E=0.02, p<.001,95 \%$ CI $[0.030 .1]$ ).

Conclusion. Using the FRAIL scale, a simple tool to screen for frailty, we identified high prevalence of frailty among PWH. Further studies are needed to identify the best tools to assess frailty and prevent poor health outcomes among this vulnerable population.

Disclosures. All Authors: No reported disclosures

954. Missed opportunities for HIV Screening in the Emergency Department Amanda Hirsch, $\mathrm{BA}^{1}$; Christopher F. Carpenter, MD, MHSA ${ }^{2}$; ${ }^{1}$ Oakland University William Beaumont School of Medicine, Royal Oak, Michigan; ${ }^{2}$ Beaumont Hospital, Royal Oak, Michigan

\section{Session: P-45. HIV: Epidemiology and Screening}

Background. There are 1.14 million people infected with human immunodeficiency virus (HIV) in the United States, and only about $86 \%$ are diagnosed. HIV diagnosis is the first step to care and expanded testing is essential to reduce transmission. Individuals with undiagnosed HIV have a transmission rate 3.5 times higher than those aware of their infection. Individuals seeking testing and treatment for sexually transmitted infections (STIs) represent a higher risk population for HIV infection. Despite revised Centers for Disease Control and Prevention (CDC) recommendations to expand HIV testing in healthcare settings, testing remains low. A significant obstacle to expanded testing, especially in emergency departments (EDs), is concern about ensuring appropriate HIV test tracking and follow-up.

Methods. We performed a retrospective chart review of patients presenting with symptoms of an STI between January 1, 2015 and July 8, 2019 at eight Beaumon Health EDs in Southeast Michigan. De-identified data was collected from the electronic health record (EHR) for patients aged 10 and older who had testing for one or more STIs including gonorrhea, syphilis, and chlamydia. Patients were evaluated for concurrent HIV testing during the encounter, and patients known to be HIV infected were excluded.

Results. Of 32,640 encounters during which patients not known to be HIV infected were tested for STIs, only $68(0.21 \%)$ included HIV antibody/antigen 
screening. Of those tested, only one $(1.47 \%)$ returned reactive. The remaining 67 screenings returned non-reactive. Applying only $10 \%$ of this diagnosis rate to the total number of STI encounters suggests an opportunity to diagnose 47 additional individuals; applying $50 \%$ of this rate and the corresponding value is 239 individuals.

Conclusion. These results highlight the need for expanded HIV screening in EDs. Systematic HIV test tracking and follow-up removes this burden from ED providers and enables expanded HIV testing in these settings.

Disclosures. All Authors: No reported disclosures

\section{Addressing Gaps and Disparities in HIV testing in the Emergency} Department

Ashley Huggett, $\mathrm{DO}^{1}$; Caroline Derrick, $\mathrm{PharmD}^{2}$; Stephanie Shealy, PharmD 3 ; Divya Ahuja, $\mathrm{MD}^{4}$; Majdi Al-hasan, $\mathrm{MD}^{2}$; Sharon Weissman, $\mathrm{MD}^{2}$; ${ }^{1}$ University of South Carolina School of Medicine/Prisma Health, Columbia, South Carolina ${ }^{2}$ University of South Carolina, Columbia, SC; ${ }^{3}$ University of South Carolina College of Pharmacy, Columbia, South Carolina ${ }^{4}$ University of South Carolina School or Medicine, Columbia, South Carolina

\section{Session: P-45. HIV: Epidemiology and Screening}

Background. Early diagnosis of HIV is key to improving outcomes for persons living with HIV (PWH). The Emergency Department (ED) is a critical site for PWH to access care. Previous studies in South Carolina (SC) have shown that two-thirds of individuals newly diagnosed with HIV have visited a health care facility a mean 7 times prior to their HIV diagnosis. Over $80 \%$ of these visits were to the ED, thus representing missed opportunities. Failure to test results from a multitude of barriers, including avoidance of testing due to a perceived lack of follow up. To address this gap in care we established a rapid HIV engagement team (RHET) that assumes responsibility for post-HIV testing linkage and reporting. The goal of this study is to evaluate the effectiveness of this intervention.

\begin{tabular}{|c|c|c|c|c|}
\hline & All $(n=4104)$ & Pre $(n=2154)$ & Post $(n=1950)$ & P-value \\
\hline Age, mean (years) & 31.4 & 31.7 & 31.0 & NS \\
\hline Male gender, $n(\%)$ & $980(23.9)$ & $489(22.7)$ & $491(25.2)$ & 0.09 \\
\hline \multicolumn{5}{|l|}{ Race/Ethnicity, n (\%) } \\
\hline White, $\mathrm{n}(\%)$ & $916(22.3)$ & $554(25.7)$ & $362(18.6)$ & $<0.001$ \\
\hline Black, n (\%) & $2885(70.3)$ & $1462(67.9)$ & $1423(73.0)$ & $<0.001$ \\
\hline Hispanic, n (\%) & $196(4.8)$ & $84(3.9)$ & $112(5.7)$ & $<0.001$ \\
\hline Other, $\mathrm{n}(\%)$ & $107(2.6)$ & $54(2.5)$ & $53(2.7)$ & 0.61 \\
\hline \multicolumn{5}{|l|}{ Reason for ED Visit, $n(\%)$} \\
\hline STI Related, $\mathrm{n}(\%)$ & $1629(39.7)$ & $848(39.4)$ & $781(40.0)$ & $<0.001$ \\
\hline Constitutional Symptoms, $\mathrm{n}(\%)$ & $66(1.6)$ & $22(1.0)$ & $44(2.3)$ & NS \\
\hline Other, $\mathrm{n}(\%)$ & $2409(58.7)$ & $1284(59.6)$ & $1125(57.7)$ & $<0.001$ \\
\hline Day of the week & & & & NS \\
\hline Sunday & $563(13.7)$ & $284(13.2)$ & $279(14.3)$ & \\
\hline Monday & $598(14.6)$ & $325(15.1)$ & $273(14.0)$ & \\
\hline Tuesday & 639 (15.6) & $359(16.7)$ & $280(14.4)$ & \\
\hline Wednesday & $590(14.4)$ & 308 (14.3) & $282(14.5)$ & \\
\hline Thursday & $589(14.3)$ & 305 (14.2) & $284(14.6)$ & \\
\hline Friday & $591(14.4)$ & $309(14.3)$ & $282(14.5)$ & \\
\hline Saturday & $534(13.0)$ & $264(12.3)$ & $270(13.8)$ & \\
\hline \multicolumn{5}{|l|}{ STI Testing Obtained, $n(\%)$} \\
\hline Gonorrhea & & $1829(84.9)$ & $1747(89.6)$ & 0.09 \\
\hline Chlamydia & & $1783(82.8)$ & $1729(88.7)$ & 0.19 \\
\hline Trichomonas & & $1518(70.4)$ & $1354(69.4)$ & 0.21 \\
\hline HSV & & $38(1.8)$ & $33(1.7)$ & 0.003 \\
\hline HIV Testing Obtained, $\mathrm{n}(\%)$ & & $131(6.1)$ & 251 (12.9) & $<0.001$ \\
\hline
\end{tabular}

Methods. This retrospective cohort study compared HIV testing rates and patterns in Prisma Health EDs from May 2018 through October 2018 (pre-RHET) to $5 / 2019$ through 10/2019 (post-RHET). Included persons were $\geq 18$ years of age and had ICD-10 codes for a sexually transmitted infection (STI), trichomonas, herpes simplex, and gonorrhea (GC) or Chlamydia (CT) NAAT, and/or presented with an initial complaint of a STI. Multivariable logistic regression analysis was utilized to examine impact of RHET implementation on HIV testing in ED.

Results. A total of 4104 individuals were identified, 2154 pre-RHET and 1950 post-RHET. Table 1 displays baseline characteristics for the two groups. Overall, $87 \%$ had GC testing; $9 \%$ had positive results; $95 \%$ had CT testing, $12.6 \%$ had positive results. Only $6 \%$ were tested for HIV pre-RHET implementation. HIV testing improved to $12 \%$ post-PHET implementation $(\mathrm{p}<0.001)$. In the multivariate regression analysis predictors for HIV testing were presenting post-RHET (OR 2.27; $95 \%$ CI 1.81 to 2.85 ), male gender (OR 2.98; $95 \%$ CI 2.39 to 3.73 ), white race (OR 2.27; 95\% CI 1.81 to 2.85), and presenting to ED for STI (OR 3.58; 95\% CI 2.036 .33 ).

Conclusion. HIV testing rates increased post-RHET yet, despite indications for HIV testing, only a small proportion received HIV testing. Further interventions are needed to improve HIV testing in EDs, particularly in women and blacks. The overall HIV testing rate remained low, representing ongoing missed opportunities for early HIV diagnosis.

Disclosures. All Authors: No reported disclosures
956. Delayed HIV diagnosis in Philadelphia

Lisa A. Spacek, MD, $\mathrm{PhD}^{1}$; Dana Higgins, $\mathrm{MPH}^{2}$; Kathleen Brady, $\mathrm{MD}^{2}$; ${ }^{1}$ Thomas Jefferson University, WYNNEWOOD, Pennsylvania; ${ }^{2}$ Philadelphia Department of Health, Philadelphia, Pennsylvania

Session: P-45. HIV: Epidemiology and Screening

Background. Ending the HIV Epidemic (EHE) requires prompt diagnosis and treatment of HIV to reduce transmission. Delayed HIV diagnosis and late entry into care remain challenging. Strategic deployment of testing resources may leverage both targeted and universal testing to accomplish the timely diagnosis of HIV infection.

Methods. We extracted data from the City of Philadelphia's Enhanced HIV/AIDS Reporting System for 3,856 individuals diagnosed with HIV infection in Philadelphia, PA from 2012-2018, to determine characteristics associated with delayed diagnosis, defined as: AIDS diagnosed within 90 days of HIV or date of AIDS diagnosis prior to HIV diagnosis. Independent variables included: time since HIV diagnosis, age category, birth sex, current gender, race/ethnicity, transmission risk, insurance status, and receipt of care from Ryan White medical provider. We used Chi-square and multivariate logistic regression to assess factors associated with delayed diagnosis.

Results. From 2012 to 2018, the number of HIV diagnoses declined from 731 to 422 ; those with delayed diagnosis declined from $28 \%$ to $18 \%$. Age category of 25-34 years comprised the majority of HIV diagnoses $\mathrm{N}=1402$ (36\%). The majority were: born male (78\%), current gender male (76\%), black (69\%), MSM (51\%), insured (54\%), and participating in Ryan White care $(71 \%)$. In multivariate regression, current gender male, heterosexual transmission, race/ethnicity Asian, American Indian, Alaska Native, or Multi-race, unknown insurance status, and receipt of care from a Ryan White medical provider were 3.7 (95\%CI, 1.2-11.4), 1.3 (1.0-1.7), 1.8 (1.2-2.8), 5.9 (4.9-7.1), and $1.4(1.2-1.7)$ times as likely to have delayed diagnosis, respectively, after adjustment for time since diagnosis, age category, and birth sex.

Participants' Characteristics and Logistic Regression Results

\begin{tabular}{|c|c|c|c|c|}
\hline Variable & $\begin{array}{l}\text { HIV (non- } \\
\text { AIDS) } \\
\text { Col \% }\end{array}$ & $\begin{array}{c}\text { AIDS } \\
\text { (Concurrent) } \\
\text { Col \% }\end{array}$ & $\begin{array}{l}\text { Logistic Regression } \\
\text { OR }(95 \% \mathrm{Cl})\end{array}$ & $\begin{array}{l}\text { Adjusted Logistic } \\
\text { Regression } \\
\text { aOR }(95 \% \text { CI) }\end{array}$ \\
\hline \multicolumn{5}{|l|}{ Time since diagnosis } \\
\hline$<3$ Years & $1121(37.3)$ & 265 (31.3) & 1.00 (Ref) & 1.00 (Ref) \\
\hline $3-4$ Years & $880(29.3)$ & $231(27.2)$ & $1.110(0.912-1.353)$ & $0.754(0.599-0.948)$ \\
\hline 4+ Years & $1007(33.5))$ & $352(41.5)$ & $1.479(1.234-1.772)$ & $0.937(0.757-1.158)$ \\
\hline \multicolumn{5}{|l|}{ Age Category: } \\
\hline $18-24$ & $384(12.8)$ & $37(4.4)$ & 1.00 (Ref) & 1.00 (Ref) \\
\hline $25-34$ & $1202(40.0)$ & $200(23.6)$ & $1.726(1.193-2.496)$ & $1.415(0.957-2.090)$ \\
\hline $35-44$ & $593(19.7)$ & $197(23.2)$ & $3.446(2.371-5.008)$ & $3.001(2.000-4.503)$ \\
\hline $45-54$ & $412(13.7)$ & $207(24.4)$ & $5.212(3.577-7.594)$ & $4.453(2.939-6.747)$ \\
\hline $55-64$ & $327(10.9)$ & $134(15.8)$ & $4.251(2.870-6.295)$ & $3.692(2.382-5.722)$ \\
\hline $65+$ & $90(3.0)$ & $73(8.6)$ & $8.414(5.326-13.29)$ & $7.004(4.189-11.710)$ \\
\hline \multicolumn{5}{|l|}{ Birth Sex } \\
\hline Male & $2331(77.5)$ & $660(77.8)$ & 1.00 (Ref) & 1.00 (Ref) \\
\hline Female & $677(22.5)$ & $188(22.2)$ & $0.981(0.817-1.178)$ & $2.556(0.821-7.955)$ \\
\hline \multicolumn{5}{|l|}{ Current Gender } \\
\hline Female & $675(22.4)$ & $183(21.6)$ & 1.00 (Ref) & 1.00 (Ref) \\
\hline Male & $2269(75.4)$ & $656(77.4)$ & $1.066(0.886-1.283)$ & 3.657 (1.178-11.353) \\
\hline Transgender & $64(2.1)$ & $9(1.1)$ & $0.519(0.253-1.062)$ & $3.174(0.870-11.573)$ \\
\hline \multicolumn{5}{|l|}{ Race/Ethnicity } \\
\hline Black & $2051(68.2)$ & 589 (69.5) & 1.00 (Ref) & 1.00 (Ref) \\
\hline White & $400(13.3)$ & $104(12.3)$ & $0.905(0.716-1.144)$ & $0.898(0.685-1.176)$ \\
\hline Hispanic & $469(15.6)$ & $113(13.3)$ & $0.839(0.670-1.051)$ & $0.894(0.696-1.150)$ \\
\hline \multirow{2}{*}{\multicolumn{5}{|c|}{$\begin{array}{l}\text { Asian/American } \\
\text { Indian/Alaska } \\
\text { Native/Multi-race }\end{array}$}} \\
\hline & & & & \\
\hline \multicolumn{5}{|l|}{ Transmission Risk } \\
\hline MSM & $1604(53.3)$ & $361(42.6)$ & 1.00 (Ref) & 1.00 (Ref) \\
\hline PWID & $245(8.1)$ & $55(6.5)$ & $0.997(0.729-1.365)$ & $0.748(0.520-1.078)$ \\
\hline MSM/PWID & $67(2.2)$ & $11(1.3)$ & $0.729(0.382-1.394)$ & $0.579(0.289-1.160)$ \\
\hline Heterosexual & $965(32.1)$ & 371 (43.9) & $1.708(1.448-2.016)$ & $1.324(1.040-1.685)$ \\
\hline No Report Risk & $127(4.2)$ & $50(5.9)$ & $1.749(1.237-2.474)$ & $1.067(0.710-1.603)$ \\
\hline \multicolumn{5}{|l|}{ Insurance Status } \\
\hline & $1,800(59.8)$ & $264(31.1)$ & 1.00 (Ref) & 1.00 (Ref) \\
\hline No & $629(20.9)$ & $88(10.4)$ & $0.954(0.737-1.234)$ & $1.082(0.829-1.411)$ \\
\hline Unknown & $580(19.3)$ & $496(58.5)$ & $5.841(4.897-6.966)$ & 5.923 (4.918 - 7.135) \\
\hline \multicolumn{5}{|l|}{ Ryan White Care } \\
\hline No/Unknown & $870(28.9)$ & $246(29.0)$ & 1.00 (Ref) & 1.00 (Ref) \\
\hline Yes & 2138 (71.1) & $602(71.0)$ & $0.996(0.842-1.178)$ & $1.416(1.1661 .719)$ \\
\hline
\end{tabular}

Conclusion. EHE will only be successful by reaching all people living with HIV and creating opportunities for early diagnosis. Routine opt-out universal screening combined with repeated, targeted testing will allow for identification and early treatment of HIV infection. As a medical care safety net, Ryan White program provides care to a disproportionate number of people with delayed diagnosis of HIV. By diagnosing HIV as early as possible, we may eliminate delayed diagnosis and reduce the risk of AIDS-related events or death.

Disclosures. All Authors: No reported disclosures

957. Efficacy of Using Disease Intervention Specialists (DIS) to Re-engage Out of Care HIV/HCV Co-Infected Persons into HCV Treatment

Maximilian D. Wegener, $\mathrm{MPH}^{1}$; Ralph P. Brooks, $\mathrm{MS}^{2}$; Suzanne Speers, $\mathrm{MPH}^{3}$; Deborah Gosselin, $\mathrm{RN}^{3}$; Merceditas Villanueva, $\mathrm{MD}^{4}$; ${ }^{1}$ Yale University School of Medicine - AIDS Program, New Haven, Connecticut; ${ }^{2}$ Yale School of Medicine - AIDS Program, New Haven, Connecticut; ${ }^{3}$ CT Department of Public Health, Hartford, Connecticut; ${ }^{4}$ Yale School of Medicine, New Haven, Connecticut 